\title{
Departure Aircraft Taxi Time Prediction Based on Gradient Boosting Regression Tree
}

\author{
Mingxin $\mathrm{GU}^{+}$, Chaoyang LU, Meize DAI and Jingjing QIAN
}

Civil Aviation College of Nanjing University of Aeronautics and Astronautics, Nanjing, China

\begin{abstract}
The rough estimation of aircraft taxi-out time causes the waste of runway slot and the low utilization efficiency. Therefore, it is urgent to propose a precise taxi time estimation method. Using the current operational data of the airport, combined with data mining to find potential factors affecting aircraft taxiing time. First, considering the large data dimensions, complexity of the algorithm and prediction inaccuracy caused by redundant learning samples, a feature selection model for optimal set of feature variables is established based on Gradient Boosted Tree (GBT). Then, the taxi-out time is predicted based on Gradient Boosting Regression Tree (GBRT). Finally, the simulation which takes Shanghai Pudong Airport as an example shows that the prediction accuracy is $76.29 \%$ within $\pm 3 \mathrm{~min}$, and up to $94.31 \%$ within $\pm 5 \mathrm{~min}$, which verifies that our method has better performance on prediction accuracy than other regression methods.
\end{abstract}

Keywords: Intelligent Traffic; Taxi Time Prediction; Gradient Boosted Tree; Departure Aircraft; Taxi-out Delay.

\section{Introduction}

Off block time (COBT) is calculated based on the calculated take-off time (CTOT) and the estimated taxiout time (EXOT). Since the EXOT is usually the average taxi-out time, it causes large errors which reduce runway availability in large airports with increasingly complex runway-taxiway structures. Therefore, how to accurately predict the taxi-out time of departure aircrafts is of great significance for the refined control decision.

Harshad Khadilkar et al.[1] presented an approach to manage the aircraft taxi-out process in a network congestion control framework. Ravizza et al.[2] proposed a multiple linear regression analysis combining both airport layout and historic taxi time data to realize the taxi time prediction. Chatterji et al.[3] introduced the surface traffic weight to predict the taxi time. Srisvastava et al.[4] established a prediction model based on multiple linear regression. Feng xia et al.[5] constructed the prediction model of waiting departure time based on the queuing theory. However, current research generally uses queuing theory or linear regression to predict the taxi time, and it is difficult to consider multiple influencing factors. Compared with aviation field, the prediction of ground transportation has been widely studied. Wang xiang[6] constructed a short-term travel time prediction model based on $\mathrm{K}$ nearest neighbor nonparametric regression in terms of traffic characteristics in different time of a day. Zhao qianyun[7] used support vector machine, random forest algorithm and other methods to predict the taxi time.

In this paper, Firstly, based on the GBT model, feature selection is performed to determine the optimal feature variable set. Secondly, the taxi time prediction model is constructed based on GBRT. Finally, the model is established for sets of data, and some common regression prediction models are compared to prove the superiority of the proposed method.

Corresponding author. Tel.: 18061693126;

E-mail address: njgumingxin@126.com. 


\section{Feature Selection Based on GBT}

\subsection{Construct an Initial Set of Feature Variables}

This paper takes the actual operation data of Pudong Airport from January 8 to November 24, 2016 as the sample data set, with a total of 173,192 lines of samples. Most of the factors affecting the taxi time of departure can be obtained according to the flight schedule. These include planned departure time $\left(T_{\text {plan }}\right)$, runway number $(r)$, airline( airline), destination( destination), registration number( REG ), flight type( $C L A)$, terminal (terminal ), stand number $(g)$, aircraft model $(C A T)$.

But some factors are uncertain so that need to be obtained through data mining. In the rush hours, departing flights may encounter conflicts during taxiing and need to wait for other flights. This paper makes a correlation analysis to find other influencing factors based on the actual operation data. For example, the number of simultaneous taxiing flights can be counted according to the number of actual and estimated departure flights 5 minutes before and after the push-off. As shown in figure 1, the correlation analysis between the taxiing time and the number of taxiing flights within the same time period is given, $R^{2}=0.7044$. Therefore, the number of simultaneous taxiing flights is included as the research scope of this paper.

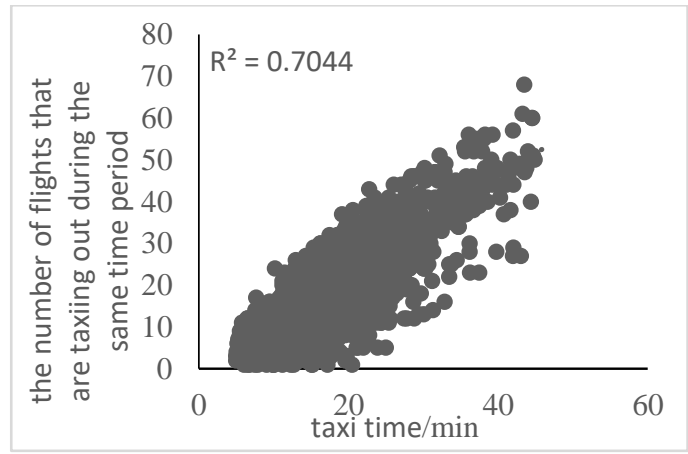

Fig. 1: The correlation between the number of flights being slipped and the taxi time at the same time

Other influencing factors include the number of simultaneous taxiing flights $\left(N_{p u s h}\right)$, the average taxi time 15 minutes before the push-off $\left(t_{\text {taxi }}\right)$, the taxi distance $(d)$, the peak situation $\left(s_{\text {peak }}\right)$ and the number of land and take-off flights during the taxiing period $\left(N_{\text {land }+ \text { takeoff }}\right)$. So, the initial set of feature variables constructed is:

$$
X^{\prime}=\left\{T_{\text {plan }}, r \text {, airline, destination, } R E G, C L A \text {, terminal }, g, C A T, N_{\text {push }}, t_{\text {taxi }}, d, s_{\text {peak }}, N_{\text {land }+ \text { takeoff }}\right\}
$$

\subsection{Feature Selection Model}

The GBT model can measure the importance of features. the greater the weight of each feature, the more important it is. The model evaluates the importance of features on each tree and calculates the average of all trees. The average is the global importance of the feature. The importance of the feature $j$ on a single tree $T_{m}$ is expressed as shown in equation (1). The global importance can be expressed as equation (2).

$$
\begin{gathered}
\operatorname{impt}_{j}^{2}\left(T_{m}\right)=\sum_{t=1}^{L-1} I_{t}^{2} 1\left(v_{t}=j\right) \\
\operatorname{impt}_{j}=\frac{1}{M} \sum_{m=1}^{M} i m p t_{j}^{2}\left(T_{m}\right)
\end{gathered}
$$

Where $M$ refers to the number of decision trees in the gradient boosting tree, and $T_{m}$ refers to a decision tree in the $M$ tree. $L$ refers to the number of leaf nodes of the decision tree $T_{m}, I_{t}^{2}$ refers to the reduction of the squared loss after the internal node $t$ splits, and $v_{t}$ refers to the feature selected when the internal node $t$ splits.

\subsection{Importance Assessment}

The importance assessment results of initial features are shown in Figure 2. The prediction accuracy is recalculated after removing each factor in ascending order. As shown in figure 3 , when the feature continues to be removed, the relative accuracy decreases continuously. Therefore, the feature of terminal is removed. 


\section{Taxiing Time Prediction Model Based on GBRT}

The core of the algorithm to predict the taxiing time is to train a basic model every time, and the following

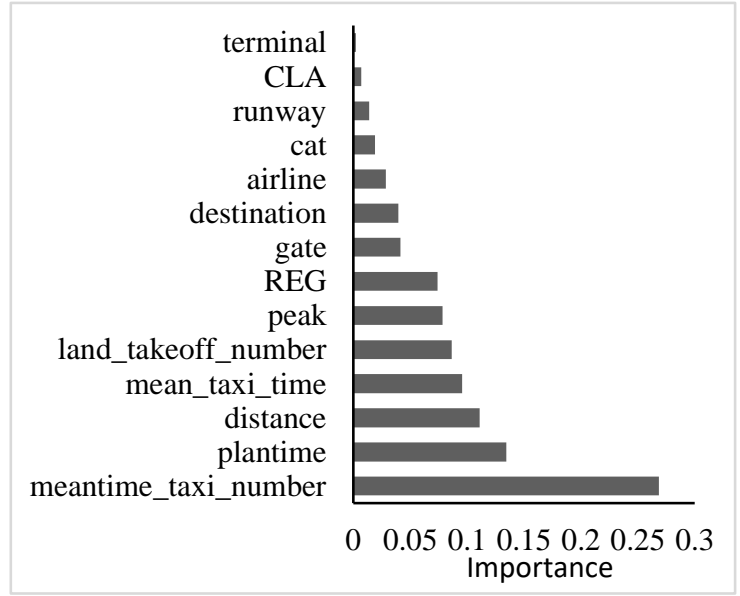

Fig. 2: Order of importance ranking

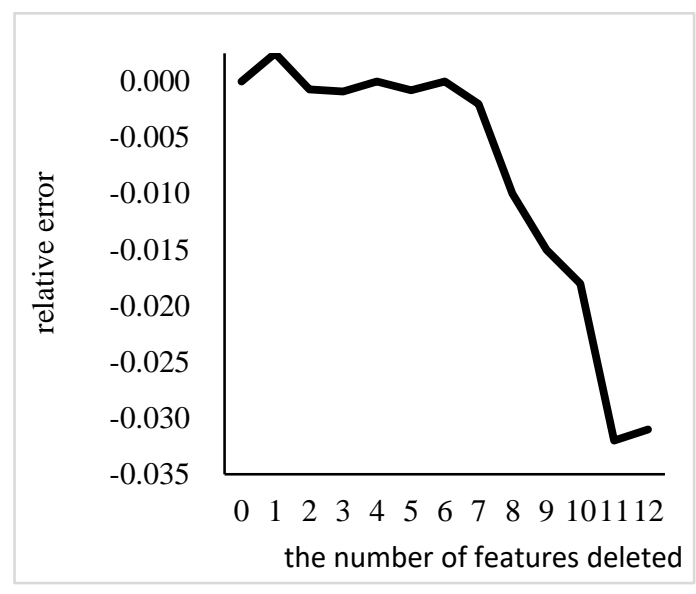

Fig. 3: Feature selection iterative process

calculation is to fit the residual error of the last model, and to establish a new model in the direction of gradient with reduced residual error according to the calculation results. The algorithm flow is as follows:

Input: training data set $T=\left\{\left(x_{1}, y_{1}\right),\left(x_{2}, y_{2}\right), \cdots,\left(x_{N}, y_{N}\right)\right\}, x_{i} \in \chi \subseteq R^{n}$ is the characteristic variable that affects the taxi time, $y_{i} \in \gamma \subseteq R$ is the taxi time; loss function $L(y, f(x)), \quad y$ represents the actual taxi time, and $f(x)$ is the predicted taxi time;

Output: Regression tree $\hat{f}(x)$ is the model that predicts the taxi time.

Firstly, initialize the model as shown in equation (3).

$$
f_{0}(x)=\arg \min _{c} \sum_{i=1}^{N} L\left(y_{i}, c\right)
$$

Secondly, for the tree $m=1,2, \cdots, M$, there are:

(1) For the number of samples $i=1,2, \cdots, N$, the formula (4) is calculated as follows.

$$
r_{m i}=-\left\lfloor\frac{\partial L\left(y_{i}, f\left(x_{i}\right)\right)}{\partial f\left(x_{i}\right)}\right\rfloor_{f(x)=f_{m-1}(x)}
$$

(2) A regression tree is fitted with $r_{m i}$ to obtain the leaf node region $R_{m j}$ of the $\mathrm{m}^{\text {th }}$ decision tree.

(3) For $j=1,2, \cdots, J$, as shown in equation (5).

$$
c_{m j}=\arg \min _{c} \sum_{x_{i} \in R_{m j}} L\left(y_{i}, f_{m-1}\left(x_{i}\right)+c\right)
$$

(4) Update

$$
f_{m}(x)=f_{m-1}(x)+\sum_{j=1}^{J} c_{m j} I\left(x \in R_{m j}\right)
$$

Where $I$ represents the indication function, when $x \in R_{m j}, I$ is equal to 1 , that is, $I$ is equal to 1 when a sample $x$ belongs to the leaf node region $R_{m j}$, otherwise it is equal to 0 .

Finally, the regression tree is output as shown in equation (7).

$$
\hat{f}(x)=f_{M}(x)=\sum_{m=1}^{M} \sum_{j=1}^{J} c_{m j} I\left(x \in R_{m j}\right)
$$




\section{Flight Delay Time Prediction Analysis}

\subsection{Experimental Data and Pretreatment}

Before data training, data is pre-processed, including the addition, deletion and standardization of the default values. The sample information after pre-processing is shown in Table 1.

\subsection{Parameter Calibration}

The square loss error is adopted as the loss function, and the parameters are set as: the number of trees $M=1000$, the default learning efficiency $L=1$, and the depth of the tree $D=3$. Accuracy $r_{\text {test }}$, error amount $e_{i}$, mean absolute error MAE and mean absolute percentage error MAPE are introduced as evaluation indicators. The expressions are shown in equations (8), (9), (10) and (11).

Table 1: Sample data after pretreatment

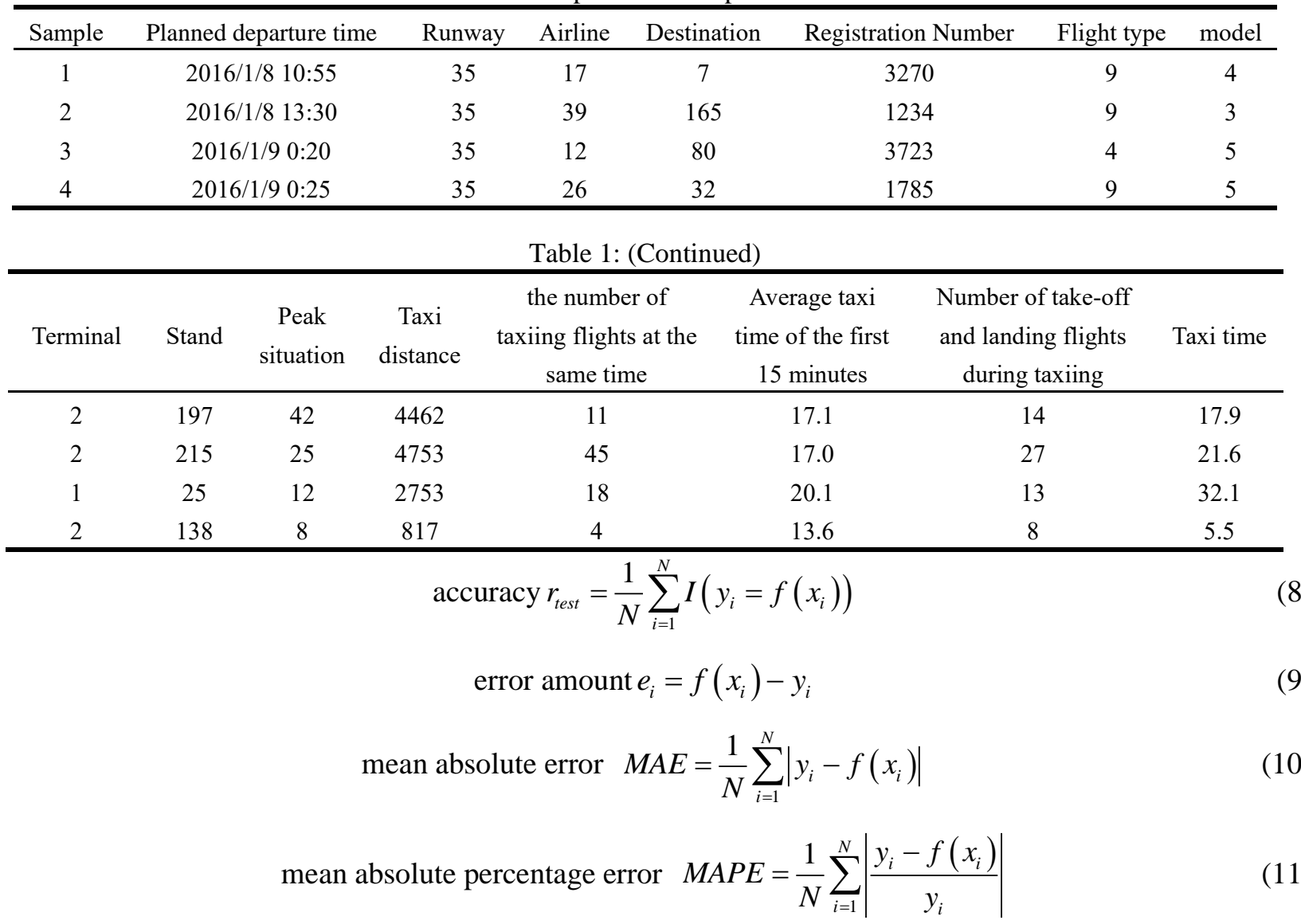

Where $N$ is the number of samples in the test set, $y_{i}$ is the actual taxi time of the test set, and $f\left(x_{i}\right)$ is the predicted taxi time of the test set.

\subsection{Taxi Time Prediction}

Through the preliminary experiment, it is found that the data of 15 days before 1 day can predict the sliding time of that day with high accuracy. This paper takes four groups of experiments as examples for multiple predictions. At the same time, four prediction methods were designed and compared, and the prediction models were established based on GBRT, linear regression (LR), elastic network regression (ETC), support vector machine regression (SVR) and Bayesian regression (BR).

Table 2 gives the prediction accuracy of the experiments using different methods, The method based on GBRT has the highest prediction accuracy. The error accuracy rate is $76.29 \%$, and the error accuracy rate is $94.31 \%$. Other prediction models are lower than the methods proposed in this paper;

Table 3 shows the MAE and MAPE results of four sets of data based on GBRT. The MAE is only 2.05min and the MAPE is only $14.46 \%$, which indicates that the prediction model can accurately predict the taxi time. 
Table 2: Accuracy of taxiing time prediction

\begin{tabular}{cccccccccccc}
\hline \multirow{2}{*}{ Set } & \multicolumn{2}{c}{ GBRT } & \multicolumn{2}{c}{ LR } & \multicolumn{2}{c}{ ETC } & \multicolumn{2}{c}{ SVR } & BR \\
\cline { 2 - 10 } & $\pm 3 \min$ & $\pm 5 \min$ & $\pm 3 \min$ & $\pm 5 \mathrm{~min}$ & $\pm 3 \mathrm{~min}$ & $\pm 5 \mathrm{~min}$ & $\pm 3 \mathrm{~min}$ & $\pm 5 \min$ & $\pm 3 \min$ & $\pm 5 \min$ \\
\hline 1 & $78.40 \%$ & $94.56 \%$ & $77.89 \%$ & $93.71 \%$ & $72.62 \%$ & $92.86 \%$ & $42.52 \%$ & $67.18 \%$ & $77.89 \%$ & $93.71 \%$ \\
2 & $76.38 \%$ & $94.30 \%$ & $71.17 \%$ & $93.32 \%$ & $68.40 \%$ & $92.67 \%$ & $40.72 \%$ & $64.98 \%$ & $71.34 \%$ & $93.32 \%$ \\
3 & $75.87 \%$ & $95.70 \%$ & $72.23 \%$ & $94.38 \%$ & $71.07 \%$ & $92.89 \%$ & $37.36 \%$ & $60.83 \%$ & $72.23 \%$ & $94.38 \%$ \\
4 & $74.51 \%$ & $92.69 \%$ & $69.97 \%$ & $90.91 \%$ & $69.64 \%$ & $92.05 \%$ & $34.74 \%$ & $55.36 \%$ & $70.13 \%$ & $91.07 \%$ \\
\hline \multirow{2}{*}{ Mean } & $76.29 \%$ & $94.31 \%$ & $72.82 \%$ & $93.08 \%$ & $70.43 \%$ & $92.62 \%$ & $38.84 \%$ & $62.09 \%$ & $72.90 \%$ & $93.12 \%$ \\
\hline
\end{tabular}

Table 3: MAE and MAPE results

\begin{tabular}{ccccc}
\hline Set & MAE/min & MAPE $/ \%$ & Average MAE/min & Average MAPE/\% \\
\hline 1 & 2.06 & $14.49 \%$ & & \\
2 & 2.03 & $14.77 \%$ & 2.05 & $14.46 \%$ \\
3 & 1.96 & $16.05 \%$ & & \\
4 & 2.16 & $12.52 \%$ & & \\
\hline
\end{tabular}

\section{Conclusions}

The aircraft taxi time prediction model based on gradient boosting regression tree is constructed. Feature selection during preprocessing improves the accuracy of the model. The model is verified by using the historical operation data of Pudong Airport, and a high accuracy rate is obtained. Compared with linear regression (LR), elastic network regression (ETC), support vector machine regression (SVR) and Bayesian regression (BR) models, the proposed method has better prediction accuracy and lower error rate.

However, this paper only considers the information provided by the standard flight schedule and the factors that can be mined from historical operational data. In the subsequent study, all possible influencing factors should be explored as far as possible, such as meteorological conditions and regulatory factors.

In addition, the method of estimating the number of taking off and landing flights during taxiing period is suitable for the case where the flow is stable. In the case of large-scale air delay, the actual number of take-off and landing flights has a large error with the calculated data. So, it is necessary to predict the number more accurately according to the actual situation, and optimize the model's characteristic variable set. This is also the content that needs to be studied in the next step.

\section{References}

[1] Khadilkar H, Balakrishnan H. Network Congestion Control of Airport Surface Operations[J]. Journal of Guidance, Control, and Dynamics, 2014, 37(3): 933-940.

[2] Ravizza S, Atkin J A D, Maathuis M H, et al. A combined statistical approach and ground movement model for improving taxi time estimations at airports[J]. Journal of the Operational Research Society, 2013, 64(9): 13471360.

[3] Chatterji G, Zheng Y. Wheels-Off Time Prediction Using Surface Traffic Metrics[C]// Aiaa Aviation Technology, Integration, \& Operations. 2012.

[4] Srivastava A. Improving Departure Taxi Time Predictions Using ASDE-X Surveillance Data[C]// Digital Avionics Systems Conference. IEEE, 2011.

[5] Feng X, Meng J S. Prediction of Flight Slip Time Based on Queuing Theory[J]. JOURNAL OF Nanjing Aerospace University, 2016, 48(5): 772-780.

[6] WANG X, CHEN X H, YANG X M. Short-term Travel Time Prediction of Expressway Based on K Nearest Neighbor Algorithm[J]. China Journal of Highway and Transport, 2015, 28(1): 102-111.

[7] Zhao Q W Research on short-term traffic flow forecast of toll station at high-speed intersection[D]. South China University of Technology, 2018. 